\title{
Metabolic reprogramming during neuronal differentiation
}

\author{
M Agostini ${ }^{1,2,6}$, F Romeo ${ }^{1,3,6,7}$, S Inoue ${ }^{4}$, MV Niklison-Chirou ${ }^{1,8}$, AJ Elia ${ }^{4}$, D Dinsdale ${ }^{1}$, N Morone $^{1}$, RA Knight ${ }^{1}$, TW Mak $^{4}$ and \\ G Melino ${ }^{*, 1,2,5}$
}

Newly generated neurons pass through a series of well-defined developmental stages, which allow them to integrate into existing neuronal circuits. After exit from the cell cycle, postmitotic neurons undergo neuronal migration, axonal elongation, axon pruning, dendrite morphogenesis and synaptic maturation and plasticity. Lack of a global metabolic analysis during early cortical neuronal development led us to explore the role of cellular metabolism and mitochondrial biology during ex vivo differentiation of primary cortical neurons. Unexpectedly, we observed a huge increase in mitochondrial biogenesis. Changes in mitochondrial mass, morphology and function were correlated with the upregulation of the master regulators of mitochondrial biogenesis, TFAM and PGC-1 $\alpha$. Concomitant with mitochondrial biogenesis, we observed an increase in glucose metabolism during neuronal differentiation, which was linked to an increase in glucose uptake and enhanced GLUT3 mRNA expression and platelet isoform of phosphofructokinase 1 (PFKp) protein expression. In addition, glutamate-glutamine metabolism was also increased during the differentiation of cortical neurons. We identified PI3K-Akt-mTOR signalling as a critical regulator role of energy metabolism in neurons. Selective pharmacological inhibition of these metabolic pathways indicate existence of metabolic checkpoint that need to be satisfied in order to allow neuronal differentiation.

Cell Death and Differentiation (2016) 23, 1502-1514; doi:10.1038/cdd.2016.36; published online 8 April 2016

Although the brain represents only about $2 \%$ of body weight, it consumes $20 \%$ of the body's energy. This high-energy demand is mainly required for restoration of the membrane gradient, after neuronal depolarization, as well as for neurotransmitter recycling, intracellular signalling and dendritic and axonal transport. ${ }^{1}$ Neurons use mainly glucose as a source of energy, which is almost entirely oxidized through sequential glycolysis and the tricarboxylic cycle (TCA) associated with oxidative phosphorylation. However, under some circumstances, such as hypoglycaemia, neurons can use glutamate and glutamine for energy production. ${ }^{2}$ The deamidation of glutamine produces glutamate, which is then oxidized via the TCA cycle, being an efficient energy generator. ${ }^{3,4}$ To date, however, relatively few reports have addressed the metabolic changes occurring during neuronal differentiation, although mitochondrial morphology and trafficking are known to vary as a function of the differentiation stage. ${ }^{5}$ In line with this, inhibition of mitochondrial protein synthesis by chloramphenicol (CAF) arrests the differentiation of neuroblastoma cell lines. ${ }^{6}$
Once neuronal progenitors exit from the cell cycle, the immature postmitotic neurons engage in a series of developmental events. ${ }^{7,8}$ Although this process is complex and highly regulated, it can be summarized in the following steps: (i) axo-dendritic polarization; (ii) migration to reach their final destination; (iii) growth of the axon and dendritic arborization; and (iv) synapse formation. Several observations indicate that both extrinsic signals and intrinsic pathways are required for the regulation of this developmental process. ${ }^{7,9}$ However, little is known about the contribution of cellular metabolism on cortical neuron differentiation. In order to investigate the metabolic changes during this process, we employed an in vitro model of terminal differentiation of cortical neurons, which has been extensively used as a model to study neuronal cell biology. ${ }^{10}$

Here we have used this model in order to investigate the role of cell metabolism, in particular energy metabolism, during neuronal differentiation. Our findings reveal a key role in the regulation of neuronal differentiation for three metabolic pathways: glycolysis, mitochondrial biogenesis, and the glutamine-glutamate pathway. In addition, we show

${ }^{1}$ Medical Research Council, Toxicology Unit, Leicester University, Leicester LE1 9HN, UK; ${ }^{2}$ Department of Experimental Medicine and Surgery, University of Rome 'Tor Vergata', Rome 00133, Italy; ${ }^{3}$ Department of Experimental and Clinical Medicine, Magna Græcia University of Catanzaro, Salvatore Venuta Campus, Catanzaro 88100, Italy; ${ }^{4}$ The Campbell Family Institute for Breast Cancer Research, Ontario Cancer Institute, University Health Network, Toronto, Ontario M5G 2C1, Canada and ${ }^{5}$ Biochemistry Laboratory IDI-IRCC, c/o Department of Experimental Medicine and Surgery, University of Rome 'Tor Vergata', Rome 00133, Italy

${ }^{*}$ Corresponding author: G Melino, Medical Research Council, Toxicology Unit, Leicester University, Lancaster Road, Leicester LE1 9HN, UK. Tel +44 (0) 1162525564 ; Fax +44 (0) 116252 5616; E-mail: gm89@le.ac.uk

${ }^{6}$ These authors contributed equally to this work.

${ }^{7}$ Current address: Istituto Fondazione Italiana per la Ricerca sul Cancro (FIRC) di Oncologia Molecolare (IFOM), Milan 20139, Italy.

${ }^{8}$ Current address: Blizard Institute of Cell and Molecular Science, Barts and the London School of Medicine and Dentistry, Queen Mary University of London, London E1 2AT, UK. Abbreviations: MS, mass spectrometry; LC, liquid chromatography; DIV, day in vitro; DON, 6-diazo-5-oxo-L-norleucine; 2-DG, 2-deoxy-D-glucose; CAF, chloramphenicol; MEF-2, myocyte enhancer factor-2; mTOR, mammalian target of rapamycin; FCCP, carbonyl cyanide 4-(trifluoromethoxy) phenylhydrazone; GSH, glutathione; GSSG, glutathione disulphide; NAC, N-acetylcysteine; GCLM, glutamate-cysteine ligase modifier subunit; Gpx1, glutathione peroxidase 1; PFKp, platelet isoform of phosphofructokinase 1; Sod1, superoxide dismutase 1; Cox2, cyclooxygenase 2; tfb2m, transcription factor B2, mitochondrial

Received 02.9.15; revised 04.2.16; accepted 22.2.16; Edited by N Bazan; published online 08.4.2016 
that PI3K-Akt-mTOR (mammalian target of rapamycin) signalling regulates mitochondrial bioenergetics and function. This is in keeping with the relevant role exerted by the PI3K-Akt-mTOR pathway in regulating dendritic morphogenesis, and accordingly, its genetic or pharmacological inhibition results in reduced dendrite size and dendritic complexity. ${ }^{11,12}$

\section{Results}

Neuronal differentiation is associated with mitochondrial biogenesis. Mitochondrial biogenesis has been linked to several physiological and pathological processes in the brain. ${ }^{13-15}$ In order to investigate whether mitochondrial biogenesis is associated with cortical neuron differentiation, we first evaluated, by real-time PCR, the relative mitochondrial DNA (mtDNA) levels at different stages of differentiation. The results in Figure 1a show that levels of mtDNA progressively and significantly increased during differentiation. To confirm that this increase in mtDNA truly reflected an increase in mitochondrial mass, we also assayed the protein levels of the different complexes of the electron transport chain (ETC). ${ }^{16}$ The western blotting in Figure $1 \mathrm{~b}$ shows that several subunits of the ETC, namely, ATP5A (mitochondrial membrane ATP synthase $F(1) F(0)$ ATP synthase or Complex V), UQCRC2 (ubiquinol-cytochrome $c$ reductase core protein II), MTCO1 (mitochondrially encoded cytochrome $c$ oxidase I), SDHB (succinate dehydrogenase complex, subunit B, iron sulphur) and NDUF0B9, increased during neuronal differentiation. Interestingly, the increase in mitochondrial biogenesis was also observed in vivo during postnatal brain development (Figures $2 a$ and b). We also observed a change in mitochondrial morphology during cortical neuron differentiation. Indeed, as shown in Figure 1c, at day in vitro 1 (DIV1) most of the mitochondria have a rounded shape and a condensed matrix, while at DIV7 there is a significant increase of mitochondria with an elongated shape and a more typical structure. ${ }^{17}$ However, these changes were not observed in vivo during postnatal brain development at the time investigated (Supplementary Figure S1a).

Several transcription factors, such as peroxisomal proliferating activating receptor $\gamma$ coactivator- $1 a$ (PGC-1a), mitochondrial transcription factor $A$ (TFAM) and nuclear respiratory factor (NRF-1/2), have a key role in the regulation of mitochondrial biogenesis. ${ }^{18}$ Therefore, we monitored the protein levels of these transcription factors along cortical neuron differentiation, and as shown in Figure 1d, TFAM and PGC-1 $a$ were indeed upregulated during the differentiation, although no differences in the expression of NRF-1 were observed (Supplementary Figure S2a). As myocyte enhancer factor-2 (MEF-2) is also involved in the regulation of mitochondrial biogenesis through its interaction with PGC- $1 a^{19}$ and has also been implicated in the regulation of neuronal differentiation, ${ }^{20}$ we investigated changes in the expression of MEF-2 in our system. Western blotting analysis shows that during neuronal differentiation the expression of MEF-2 is indeed increased (Figure 1d). However, the expression of structural protein such as cyclophilin D does not change during neuronal differentiation as shown in Supplementary Figure S2b.

To determine the importance of PGC-1a and NRF-1, we have also tested their transcriptional activity by assessing the mRNA levels of their target genes. ${ }^{21,22}$ As shown in Figures $1 e$ and $f$, cortical neuron differentiation was also associated with an increase in the mRNA expression of glutathione peroxidase 1 (Gpx1) and transcription factor B2, mitochondrial (Tfb2m).

Previously, it has been show that inhibition of mitochondrial protein synthesis prevents cell differentiation. ${ }^{6}$ To investigate whether this was also the case for cortical neurons, DIV1 cortical neurons were treated with CAF at a concentration that does not induce cell death (Supplementary Figure S3c). Supplementary Figures S2c and d show that inhibition of mitochondrial protein synthesis led to a significant (about $58 \%$ ) reduction of neuronal differentiation, with the neurons having an arrested morphology characteristic of stage 3-4 according to Dotti et al. ${ }^{10}$ classification.

Together, these results indicate that mitochondrial biogenesis occurs during cortical neuron differentiation and most likely to be regulated by TFAM, PGC-1 $a$ and MEF-2.

Mitochondrial function and cortical neuron differentiation. The observed mitochondrial morphological changes associated with neuronal differentiation led us to investigate whether mitochondrial bioenergetics was also affected during the differentiation of cortical neurons. To do this, we used the extracellular Flux Analyser that allows the measurement of the oxygen consumption rate (OCR, mitochondrial respiration) and the extracellular acidification rate (ECAR, glycolysis) in real time. We found that the basal respiration of fully differentiated cortical neurons (DIV7) was higher than that of immature cortical neurons (DIV1) as shown in Figure 3a (before oligomycin was added). Then we assessed the function of individual ETC complexes in DIV7 and DIV1 cortical neurons by sequentially adding pharmacological inhibitors of the respiratory chain. When oligomycin was added, a decrease in OCR occurred in both undifferentiated and differentiated cortical neurons to approximately the same extent (Figure $3 \mathrm{~b}-\mathrm{i}$ ). This suggests that the mitochondrial oxygen consumption used for ATP synthesis does not change during cortical neuron differentiation. Moreover, the remaining OCR can be ascribed to proton leakage, which, as shown in Figure $3 b-i i$, is not affected by the differentiation. Next we determined the maximal OCR that the cells can sustain by adding carbonyl cyanide 4-(trifluoromethoxy) phenylhydrazone (FCCP). This treatment resulted in a stimulation of the OCR, which was significantly higher in the differentiated cells as shown in Figure 3b-iii. FCCP did not increase the OCR in the undifferentiated cells above the basal rate, and unlike the differentiated neurons, the undifferentiated cells have no spare respiratory capacity (Figure 3b-iv). In differentiated cells, the OCR is significantly increased by the uncoupler by about $60 \%$. Finally, we added Rotenone, which dramatically suppressed the OCR. No differences were observed in non-mitochondrial respiration (Figure $3 b-v)$. Moreover, ECAR, which provides a quantification of glycolytic flux, was only present at DIV7 cortical 


\section{Relative mtDNA levels}

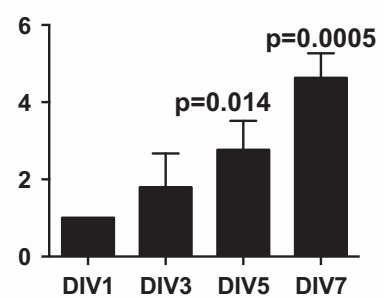

b

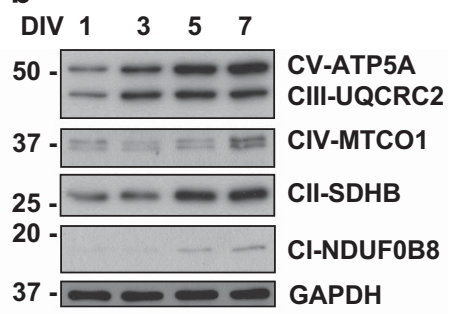

c

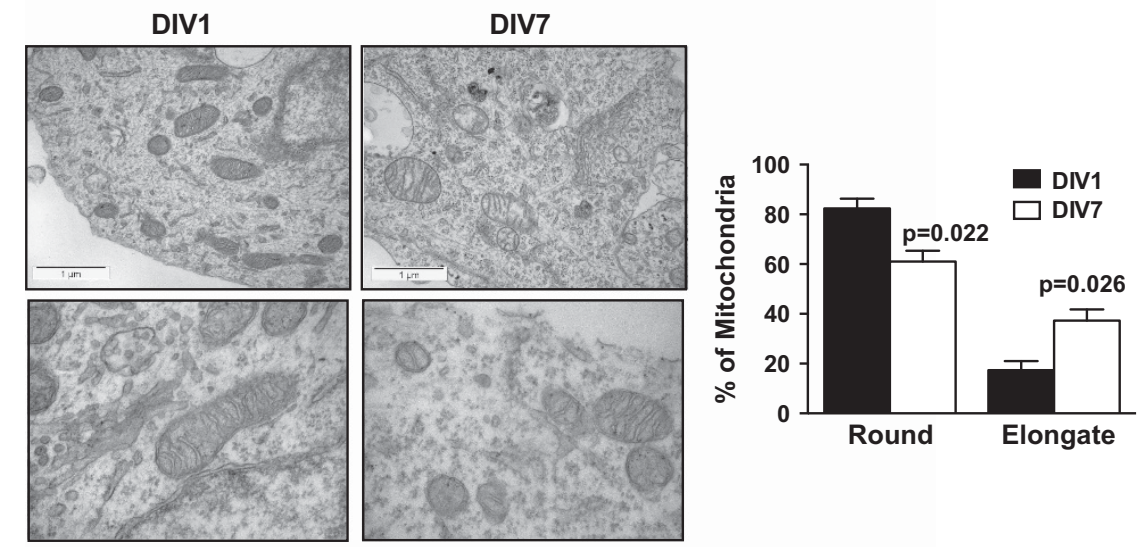

d
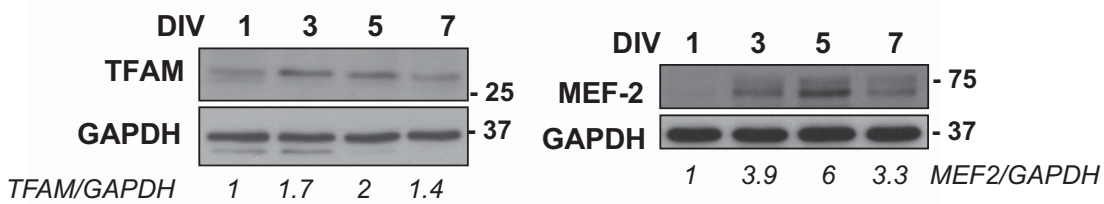

PGC-1 $\alpha$

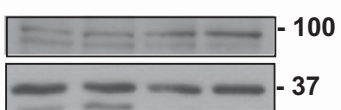

GAPDH

$\begin{array}{lll}1.1 & 1.9 & 2.1\end{array}$
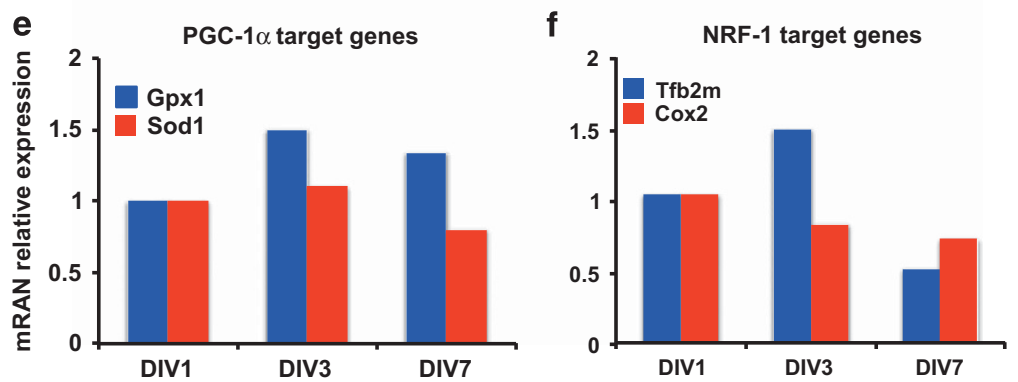

Figure 1 In vitro terminal differentiation of cortical neurons is associated with mitochondrial biogenesis. (a) Relative quantification of mtDNA copy number during differentiation of cortical neurons. Real-time PCR was performed with primers against a single-copy nuclear gene succinate dehydrogenase complex, subunit A and the mitochondrially encoded NADH dehydrogenase 5 gene. (b) Expression of the different complexes of ETC increases along the differentiation. Western blotting was performed with MitoProfile Total OXPHOS. (c) Transmission electron microscopy analysis of DIV1 and DIV7 neurons. The mitochondria at DIV1 are small and rounded, with a dense matrix. At DIV7, the mitochondria have a much less dense matrix and the percentage of mitochondria with elongated shape increase, as shown in the graph (right panel). For comparison, we restricted imaging to the perinuclear/Golgi region. (d) Western blotting analysis of the transcription factors TFAM, PGC-1 $\alpha$, MEF-2 and NRF-1 during in vitro terminal differentiation of cortical neurons. Numbers indicate densitometric analysis of a representative experiment. (e and $\mathbf{f})$ RNA levels of the indicated PGC-1 $\alpha$ and NRF-1 target genes during in vitro terminal differentiation of cortical neurons, respectively. RNA levels were assessed by real-time PCR

neurons, confirming the increase of glycolysis during differentiation (Figures $3 \mathrm{c}$ and $4 \mathrm{a}$ ).

Overall, these findings show that cortical neuronal differentiation is associated with a functional reprogramming of mitochondria.
Global metabolic profiling during in vitro neuronal differentiation. Then we asked whether other metabolic changes were associated with the mitochondrial biogenesis. To do so, cortical neurons were isolated from E16 embryos and allowed to terminally differentiate in vitro as previously 

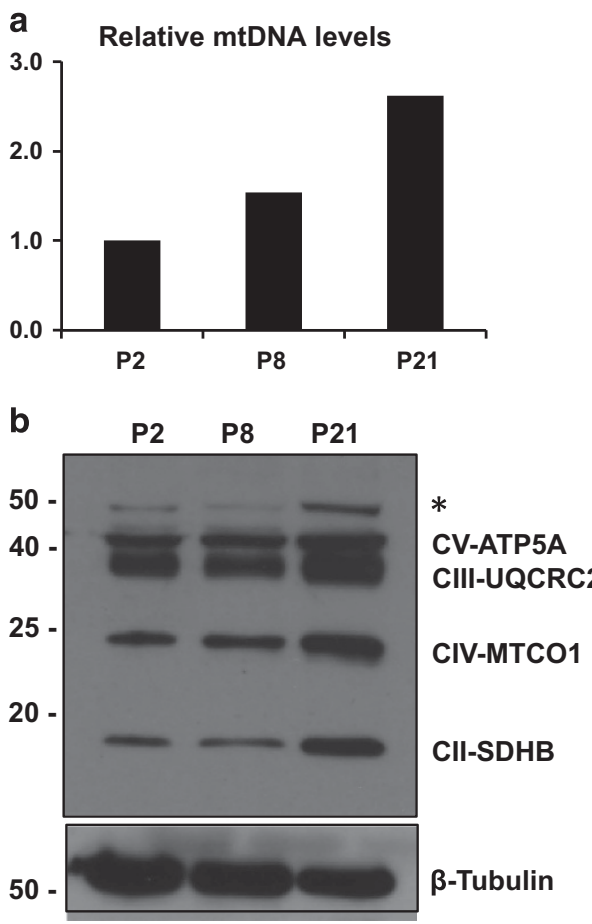

Figure 2 Mitochondrial biogenesis during postnatal development of murine cerebral cortex. (a) Relative quantification of mtDNA copy number at the indicated postnatal day. Real-time PCR was performed with primers against a single-copy nuclear gene succinate dehydrogenase complex, subunit $A$ and the mitochondrially encoded NADH dehydrogenase 5 gene. (b) Expression of the different complexes of ETC increases along the postnatal development of cerebral cortex. Western blotting was performed with MitoProfile Total OXPHOS. A representative experiment is shown. $P$, postnatal day. *Aspecific

described. $^{23}$ Cells were harvested for global metabolic profiling at DIV2, DIV5 and DIV7 to identify changes associated with differentiation. A large number of biochemical changes, associated with glycolysis, lipid metabolism, synthesis of neurotransmitters and cellular stress, were observed during differentiation of cortical neurons (Supplementary Table S1).

First, an increase in glucose metabolism was observed with an accumulation of glycolytic metabolites, such as glucose-6phosphate, glycerate, 2-phosphoglycerate, phosphoenolpyruvate and lactate between DIV2 and DIV7 (Figure 4a). These changes in classical glycolysis were accompanied by an increase in the pentose phosphate pathway (PPP) intermediates, 6-phosphogluconate and sedoheptulose-7-phosphate. Thus these data suggest that glucose oxidation increases during cortical neuron differentiation to produce biosynthetic precursors and NADPH-reducing equivalents for the synthesis of amino acids and lipids (Supplementary Table S1). We also observed an increase in long-chain fatty acids with an increase in lysolipids, whereas medium-chain fatty acids decreased, over time (Supplementary Table S1). Together with the observed increase in citrate and cholesterol synthesis and the lack of acyl-carnitine conjugates, these data suggest increased fatty acid synthesis and membrane remodelling (Supplementary Table S1). In addition, and consistent with the fully differentiated neuronal phenotype at DIV7, the concentration of several neurotransmitters (glycine, serine, glutamate and GABA) significantly increased over time in cultured neurons (Supplementary Table S1). The precursors of dopamine and serotonin, tyrosine and tryptophan, respectively, also increased significantly.

Glucose metabolism increases during differentiation of cortical neurons. Glucose is the main source of energy in the brain. ${ }^{24}$ The global metabolic profile indicates that glycolytic activity is increased during in vitro differentiation of cortical neurons. The rate of glycolysis is tightly regulated, in particular by three key enzymes: hexokinase, phosphofructokinase-1, and pyruvate kinase (Figure 4b). Therefore, we next asked whether the expression of these enzymes was increased during cortical neuron differentiation in parallel with the increased glycolytic flux. Total RNA was extracted at different days and the expression of these enzymes was monitored by real-time PCR. As shown in Supplementary Figure S3a, PFKp expression was significant increased over time although no significant changes in HK-1 and PKM expression were observed. Increase of PFKp expression was also confirmed at protein levels (Figure 4c). Moreover, we also found increased expression at the mRNA level of the glucose transporter 3 (GLUT3; neuron specific, Figure 4d). The increased GLUT3 expression would be expected to increase glucose uptake. To test this, we incubated cortical neurons with the fluorescent deoxyglucose analogue and the fluorescence intensity was evaluated by flow cytometry. As shown in Figure $4 \mathrm{e}$, the mean fluorescence intensity is significantly increased, indicating an increase of glucose uptake early in differentiation.

To test whether glucose metabolism is required for neuronal differentiation, DIV1 cortical neurons were treated with 2-deoxy-D-glucose (2-DG) a competitive inhibitor of glycolysis. No apoptosis was detected at the concentration used (Supplementary Figure S3c). As shown in Figures $4 \mathrm{f}$ and $\mathrm{g}$, cortical neuron differentiation was inhibited by roughly $75 \%$ in the presence of 2-DG, and neurons were arrested in stage 2 of the differentiation. Moreover, inhibition of glucose metabolism led to a significant reduction (about $30 \%$ ) in the endogenous levels of ATP (Figure 4h).

These results indicate that the upregulation of glucose metabolism during cortical neuron differentiation is linked to an increase in GLUT3 and PFKp expression with corresponding increases in glucose uptake and glycolytic flux and this enhanced glucose metabolism is required for the full differentiation of cortical neurons.

Glutamine-glutamate metabolism and differentiation of cortical neurons. Glutamate is the main excitatory neurotransmitter in the brain. $^{2}$ However, it has many other functions, including its oxidation for energy production (through its oxidative deamination to $a$-ketoglutarate, formation of GABA and glutathione (GSH) synthesis (Figure $5 a$ ). The global metabolic profile analysis shows that glutamate and GABA are significantly upregulated during differentiation of cortical neurons while no differences are observed in glutamine levels (Figure 5b and Supplementary Table S1). This prompted us to investigate the expression of several enzymes involved in the pathway depicted in Figure $5 \mathrm{a}$. 
a

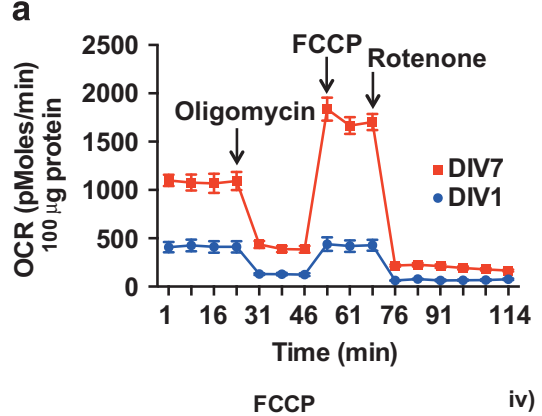

iii) (Maximal Respiration)

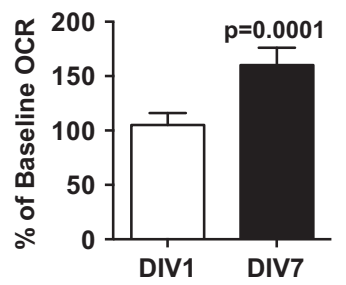

b

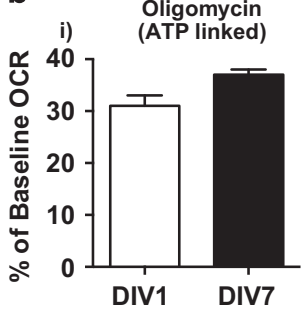
Spare Respiratory Capacity

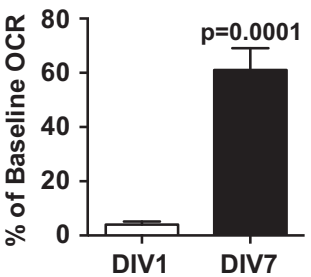

ii) Proton Leak

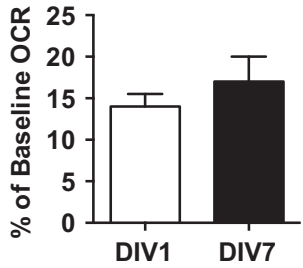

v) Rotenone (Non-Mitochondrial)

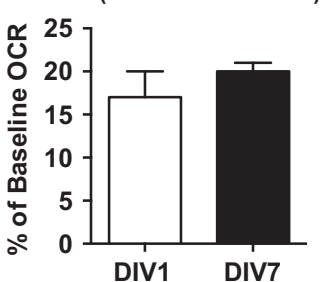

C

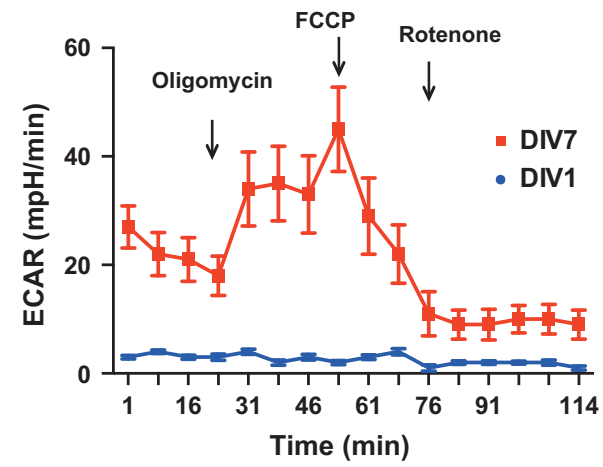

Figure 3 Mitochondrial bioenergetics analysis during in vitro terminal differentiation of cortical neurons. (a) Real-time analysis of OCR in cortical neurons at DIV1 and DIV7. ATP synthase inhibitor oligomycin, mitochondrial uncoupler FCCP and mitochondrial complex I inhibitor Rotenone were injected sequentially at the indicated time points into each well after baseline rate measurement. Fully differentiated cortical neurons (DIV7) show higher basal OCR then undifferentiated neurons (DIV1). A representative experiment of four independent is shown. (b) (i) ATP-linked; (ii) proton leak and (iii) maximal respiration is higher in fully differentiated neurons when compared with the undifferentiated neurons. At DIV7, cortical neurons have spare respiratory capacity that is absent in DIV1 cortical neurons (iv); (v) non-mitochondrial respiration. Each group is shown as a percentage of baseline (measurement before oligomycin injection). (c) ECAR is present only at DIV7 and not at DIV1. ECAR were measured using the XF24 Analyser (see Materials and Methods section for details). A representative experiment of four independent is shown. Values are mean \pm S.D. (A representative of three independent experiment is shown. Each point, $n=5$ technical replicates)

In neurons, glutamate is mainly produced by the deamidation of glutamine by glutaminase 1 (GLS1) and 2 (GLS2). ${ }^{25}$ We therefore monitored the expression of these enzymes during cortical neuronal differentiation. The mRNA levels of GLS1 and GLS2 are significantly increased from DIV3 to DIV7 as assessed by real-time PCR (Figure 5c). However, upregulation of GLS2 mRNA was not associated with an increase at protein levels (Supplementary Figure S3b). Endogenous glutamate can either feed the TCA cycle or be converted into GABA. Glutamate enters the TCA cycle after conversion into $a-K G$ by glutamate dehydrogenase 1 (GLUD1). As shown in Figure $5 \mathrm{~d}$, expression of GLUD1 mRNA is also increased during differentiation of cortical neurons. Increased GLUD1 expression (and hence increased $a-K G$ ), results in an increase in some downstream TCA metabolites, such as citrate and malate Supplementary Table S1). Finally, glutamate can also be converted into GABA, by the action of glutamic acid decarboxylase (GAD). Western blotting analysis (Figure 5e) shows that expression of GAD67, one of the two GAD isoforms expressed in brain, increases during neuronal differentiation.

To assess the biological importance of glutamine metabolism in neuronal differentiation, we treated DIV1 cortical neurons with 6-diazo-5-oxo-L-norleucine (DON), ${ }^{26}$ a selective inhibitor of GLS. DON treatment resulted in a significant reduction of neuronal differentiation (Figures $5 f$ and $g$ ), with neurons showing stages 3-4 morphology. In addition, intracellular levels of ATP were reduced (Figure 5h).

Overall, these results indicate that glutamine-glutamate metabolism is increased during the differentiation of cortical neurons and its inhibition reduces cortical neuron differentiation as well as endogenous levels of ATP.

The PI3K-Akt-mTOR pathway regulates mitochondrial bioenergetics. Several reports have shown that mTOR signalling controls neuronal development and plasticity. ${ }^{27}$ 
a
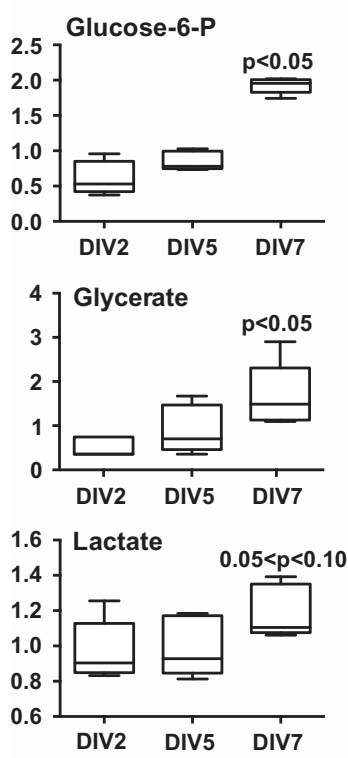

f

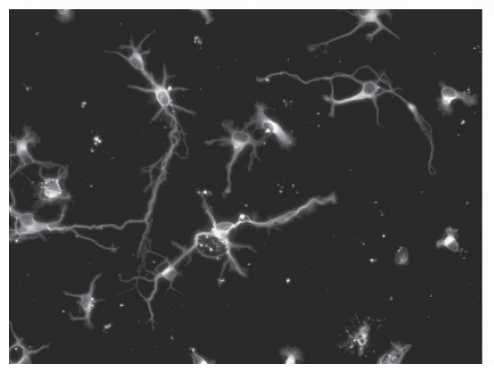

b
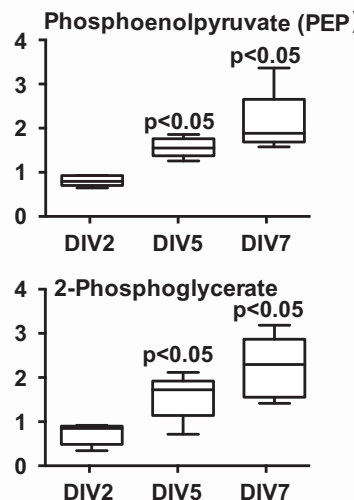

e

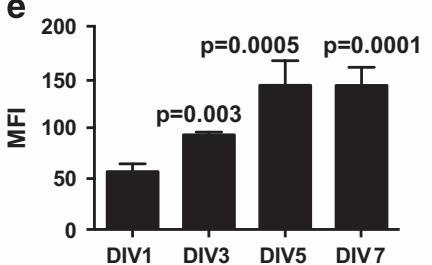

2-DG

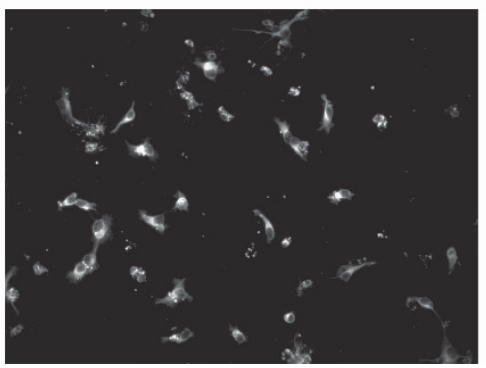

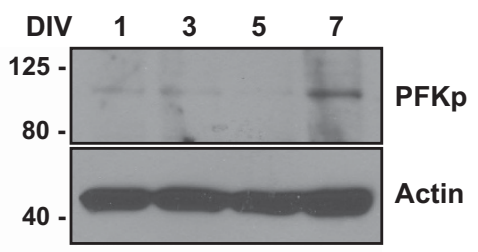
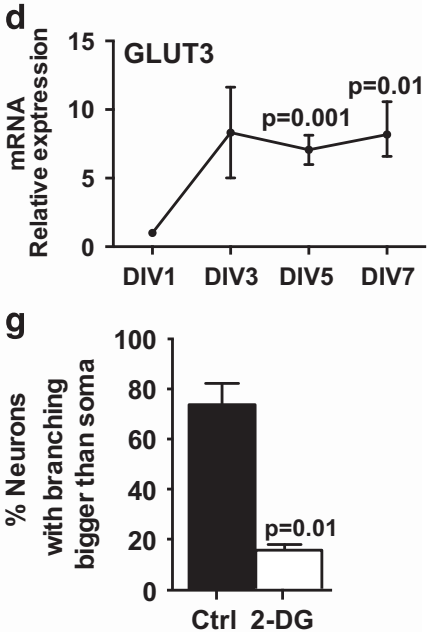

h

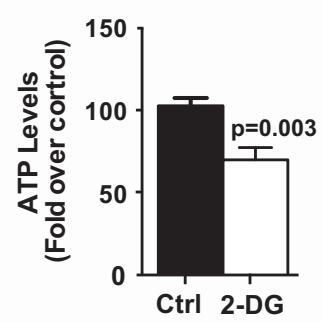

Figure 4 Glucose metabolism increases during in vitro terminal differentiation of cortical neurons. (a) Glucose metabolism is increased in differentiating neurons as indicated by increases of glycolytic metabolites over time. Levels of the indicated metabolites were evaluated as described in Materials and Methods section. Welch's two-sample $t$-tests were used to identify biochemicals that differed significantly between experimental groups ( $n=5$ for each time point). (b) Glycolytic pathway with the assayed glycolytic genes in red. (c and d) Expression of glycolytic genes during in vitro terminal differentiation of cortical neurons. Protein levels of phosphofructokinase-1 (platelet isoform PFKp, neuron specific) and mRNA levels of glucose transporter 3 (GLUT3) increase during differentiation of cortical neurons. Enzymes levels were evaluated by real-time PCR. Data are normalized to the housekeeping GAPDH and relative to DIV1. Note that the enzymes investigated are brain and neuron specific. (e) Glucose uptake of cortical neurons at the indicated stages of differentiation was assayed with 2-NBDG by FACS analysis. (f) Pharmacological inhibition of glycolysis negatively affect cortical neuron differentiation. Cortical neurons were plated at DIV1 and untreated (CTRL) or treated with 2-DG (10 mM) for $48 \mathrm{~h}$. Representative micrographs of DIV3 cortical neurons stained with $\beta$-III-Tubulin are shown. (g) Quantification of neuronal differentiation as in Figure 1f. (h) ATP levels in DIV6 cortical neurons treated for 24 h. Data represent mean \pm S.E.M. $(n=3-4$ of independent experiments; Student's t-test). MIF, mean fluorescence intensity

Moreover, the mTOR pathway has a key role in the regulation of cellular metabolism in non-neuronal tissues. ${ }^{28}$ Therefore, we asked whether mTOR also has a role in the metabolic changes observed during neuronal differentiation. To do this, DIV1 cortical neurons were treated with rapamycin, and as expected, rapamycin treatment resulted in a reduction of phosphorylated S6K (Figure 6a) together with a reduction in neuronal differentiation (Figure 6b). More importantly, mitochondrial biogenesis (Figure 6d) as well as the mitochondrial maximal respiration rate and the spare respiratory capacity (Figures $6 e$ and f) were also significantly reduced after rapamycin treatment. The PI3K/Akt-mTOR pathway cooperates with the MAPK/ERK signalling to regulate dendrite complexity. ${ }^{11}$ Therefore, to better characterize the molecular pathways involved and the relative contribution of each of them to the metabolic changes observed during neuronal differentiation, DIV1 cortical neurons were treated with the selective pharmacological inhibitors at concentrations that do not induce cell death (Supplementary Figure S3c). Inhibition of PI3K by LY294002 led to a reduction of pS6 phosphorylation levels as well as a reduction in the endogenous levels of MEF2 and TFAM as shown in Figure $6 \mathrm{c}$ that was associated with a significant reduction of mitochondrial mass as shown by the relative levels of mtDNA (Figure $6 \mathrm{~d}$ ). The same effects were observed when cortical neurons were treated with PD98059, a MAPK pathway inhibitor. In addition, at the functional level, as shown in Figure 6e, LY294002 significantly reduced the maximal respiration rate and spare 
a

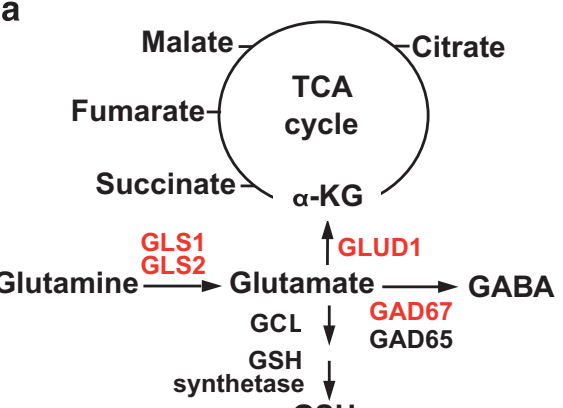

C
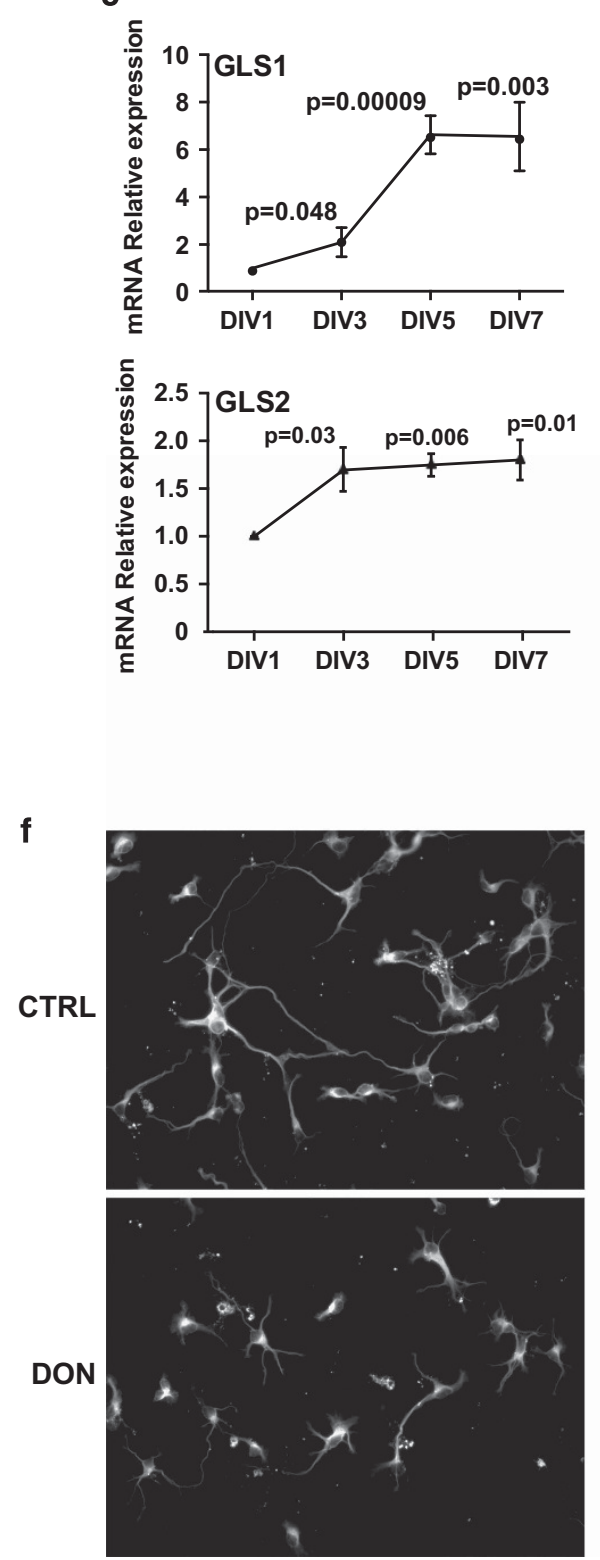

b

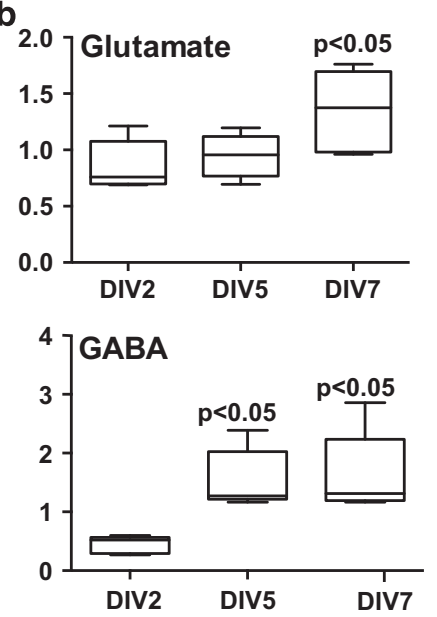

d
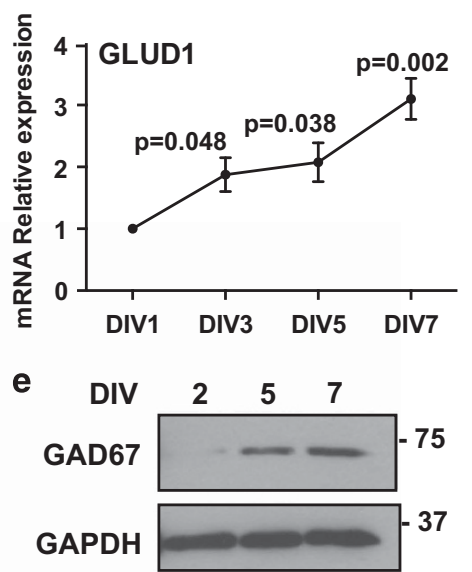

g

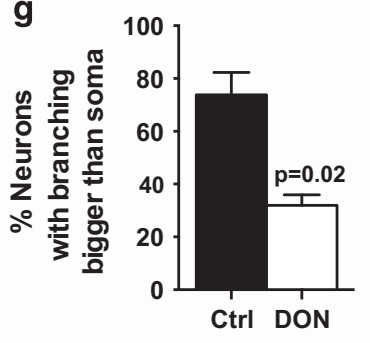

h

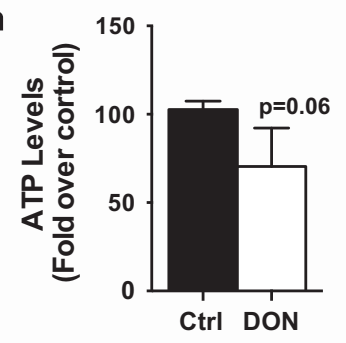

Figure 5 Glutamine-glutamate cycle increase during in vitro terminal differentiation of cortical neurons. (a) A schematic representation of Glutamine-glutamate pathway in cortical neurons. In red, the genes assayed. (b) Glutamate and GABA levels increase during cortical neuron differentiation. Levels of the indicated metabolites were evaluated as described in Materials and Methods section. Welch's two-sample t-tests were used to identify biochemicals that differed significantly between experimental groups ( $n=5$ for each time point). (c and d) mRNA levels of glutaminase type 1 (GLS1), type 2 (GLS2) and glutamate dehydrogenase 1 (GLUD1) increase during differentiation of cortical neurons. Enzyme levels were evaluated by real-time PCR. Data are normalized to the housekeeping GAPDH and relative to DIV1. (e) Western blotting analysis of glutamate decarboxylase 67 (GAD67) along the differentiation of cortical neurons (f) Pharmacological inhibition of glutamine-glutamate pathway negatively affect cortical neuron differentiation. Cortical neurons were plated at DIV1 and untreated (CTRL) or treated with DON $(10 \mu \mathrm{M})$ for $48 \mathrm{~h}$. Representative micrographs of DIV3 cortical neurons stained with $\beta$-III-Tubulin are shown. (g) Quantification of neuronal differentiation as in Figure 1f. (h) ATP levels in DIV6 cortical neurons treated for $24 \mathrm{~h}$. Data represent mean \pm S.E.M. ( $n=3$ of independent experiments; Student's $t$-test) 
b

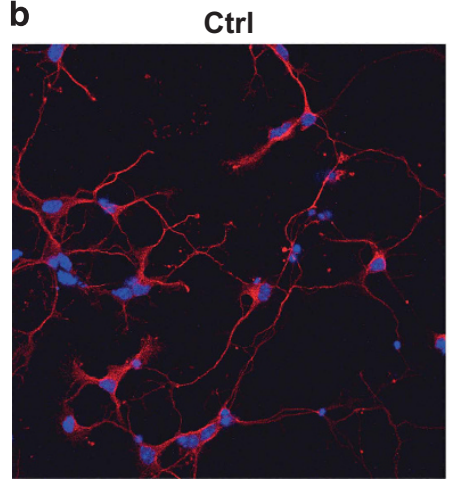

d

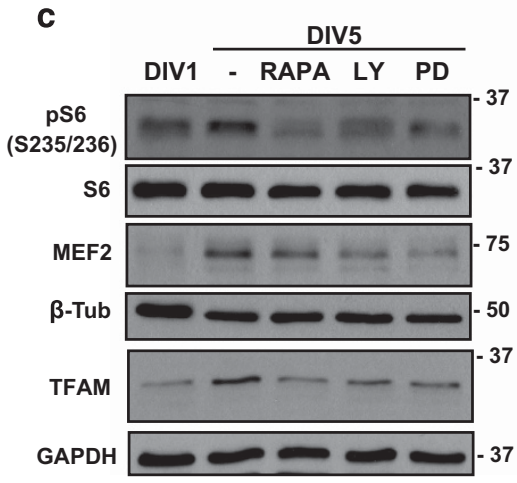

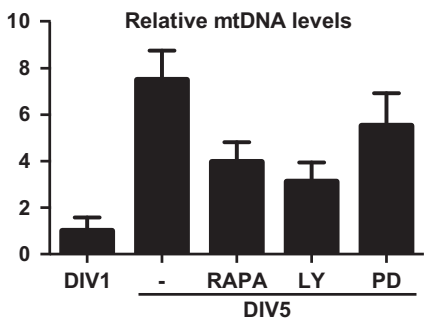

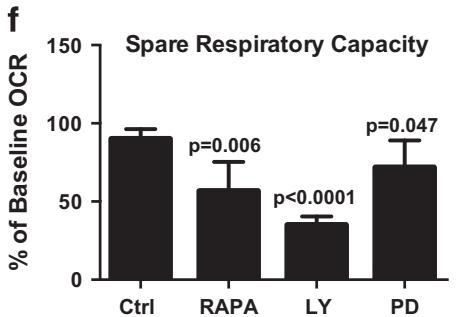

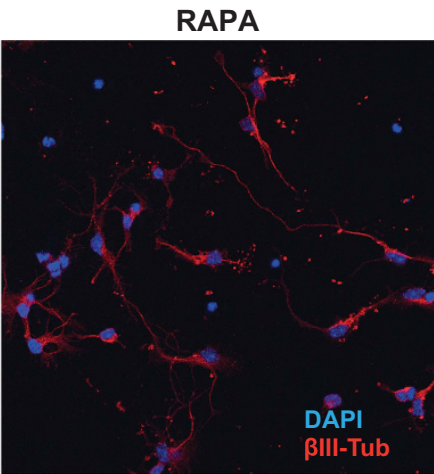

FCCP
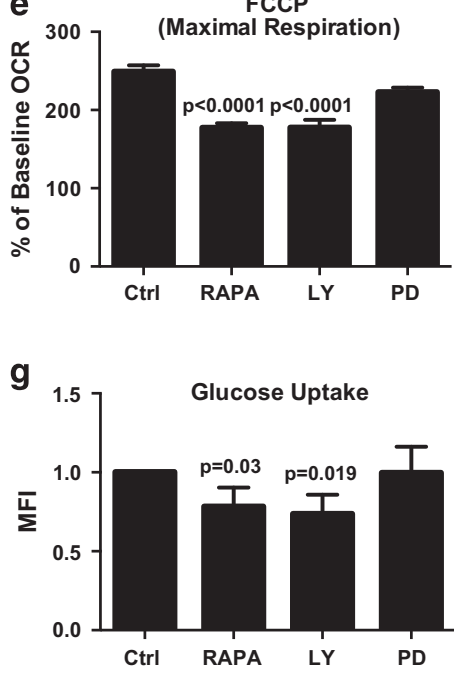

Figure 6 PI3K-Akt-mTOR signalling regulates mitochondrial bioenergetics and glucose uptake. (a) Immunoblot of S6K phosphorylation in control (Ctrl) and rapamycintreated (RAPA) cortical neurons. A representative experiment is shown $(n=3)$. (b) A representative confocal images of cortical neurons at DIV5 after rapamycin treatment showing a reduction of neuronal differentiation. After treatment, neurons were fixed and immunostained with $\beta$-III-Tubulin and DAPI. (c) A representative western blotting showing the levels of the indicated proteins. DIV1 cortical neurons were treated as indicated (RAPA = rapamycin $10 \mathrm{nM}$; LY294002 $=50 \mu \mathrm{M} ; \mathrm{PD} 98059=25 \mu \mathrm{M})$ and harvested at DIV5. (d) Relative levels of mtDNA analysed by real-time PCR of cortical neurons treated as in panel (c). (e and f) Mitochondrial maximal respiration and spare respiratoty capacity is regulated by the PI3K-Akt-mTOR pathway. Cortical neurons treated as in panel (b) were analysed using the XF24 Analyser (see Materials and Methods section for details). (g) PI3K-Akt-mTOR pathway is involved in the regulation of glucose uptake. Data represent mean \pm S.E.M. ( $n=3$ of independent experiments; Student's $t$-test)

respiratory capacity. However, maximal respiration rate and spare respiratory capacity were only marginally affected by PD98059 (Figures 6e and f). These results suggest that the $\mathrm{PI3K}$ and MAPK pathways act together in the regulation of mitochondrial biogenesis, whereas mitochondrial function is mainly regulated by PI3K-Akt-mTOR. As glucose uptake was affected by rapamycin treatment (Figure $6 \mathrm{~g}$ ), we have also assessed the uptake of glucose in the same experimental context. As shown in Figure $6 \mathrm{~g}$, PI3K inhibition resulted in a significant reduction of glucose uptake, whereas the inhibition of the MAPK pathway did not.

Role of reactive oxygen species (ROS) in neuronal differentiation. Several in vitro approaches suggest that ROS generation and signalling are involved in neuronal differentiation. ${ }^{29}$ Thus we asked whether ROS have a role in ex vivo differentiation of primary cortical neurons. Metabolic analysis shows a significant increase in glutathione disulphide (GSSG) and a slight increase in $\mathrm{GSH}$ during neuronal differentiation. Overall, this leads to a reduction in GSH/GSSG ratio that fails to reach the significance (Figures $7 \mathrm{a}-\mathrm{c}$ ). In addition, these changes were associated to an increase in ROS production during neuronal differentiation as shown in Figure $7 d$ and Supplementary Figure S4b. To explore whether this ROS production was involved in neuronal differentiation, DIV1 cortical neurons were treated with $\mathrm{N}$-acetylcysteine (NAC), ${ }^{30}$ a ROS scavenger, and neuronal differentiation was assessed by the expression of Synapsin 1-2. As shown in Figure 7e, reduction of ROS levels led to a reduction of neuronal differentiation, suggesting that ROS participate in the regulation of cortical neuron differentiation.

Next we asked whether reduced GSH synthesis affect neuronal differentiation. Hence, we employed mice lacking of the glutamate-cysteine ligase modifier subunit (GCLM), a component of the glutamate cysteine ligase (GCL), the rate-limiting enzyme of GSH synthesis (Supplementary Figure S4a), which have reduced GSH levels in all organs. ${ }^{31}$ 
a

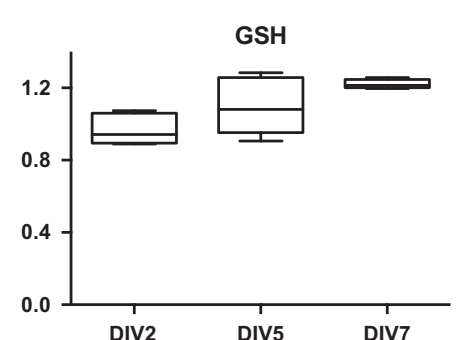

\section{b}

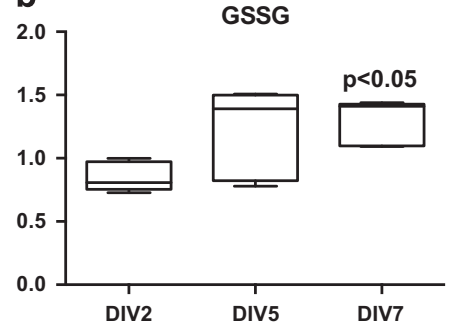

C

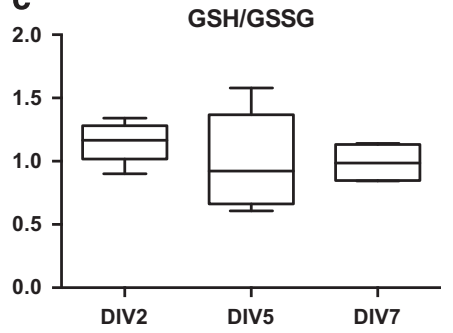

d $600 \quad \mathrm{CM}-\mathrm{H}_{2}$ DCFDA

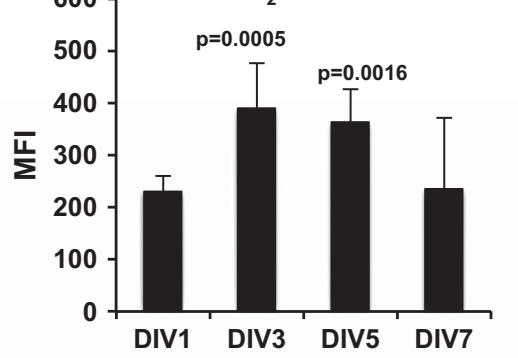

e

DIV3

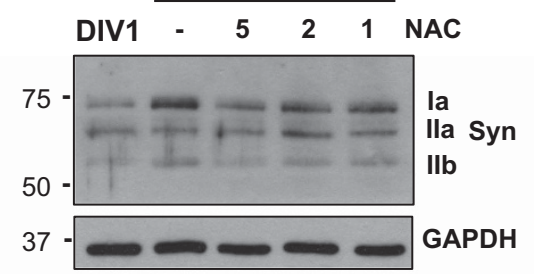

f

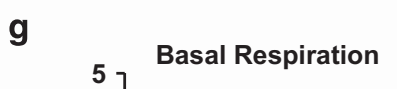

h
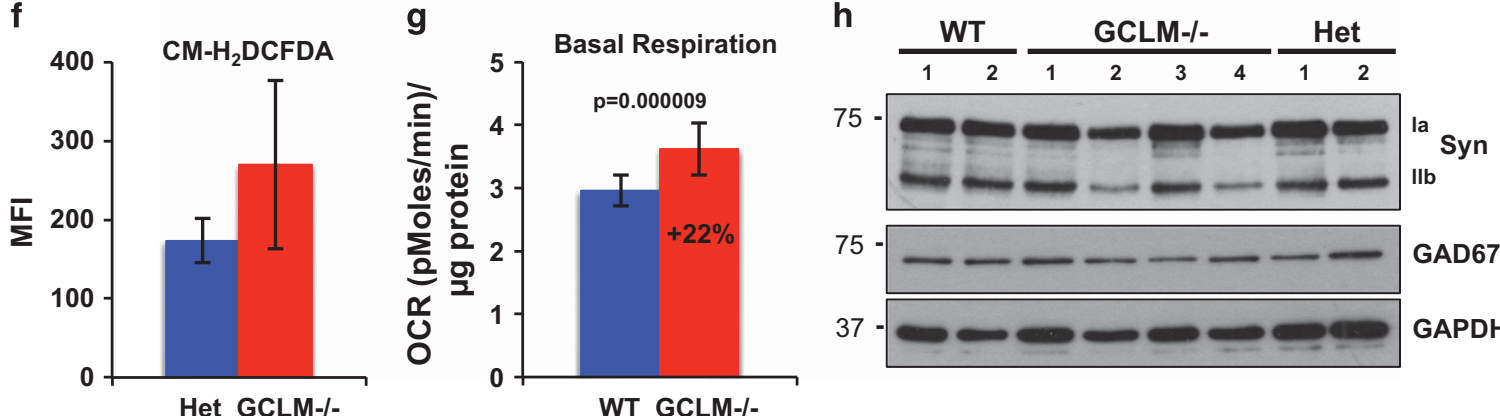

GAD67

GAPDH

Figure 7 Role of ROS in terminal neuronal differentiation. (a-c) GSSG levels increase during cortical neurons differentiation. Levels of the indicated metabolites were evaluated as described in Materials and Methods section. Welch's two-sample $t$-tests were used to identify biochemicals that differed significantly between experimental groups ( $n=5$ for each time point). (d) ROS levels during cortical neuron differentiation were assayed with CM-DCFDA by FACS analysis, Values are mean \pm S.D. ( $n=3$ independent experiments). (e) Inhibition of ROS levels by NAC ${ }^{30}$ negatively affect neuronal differentiation evaluated by Synapsin expression. (f) ROS levels in DIV7 cortical neurons derived from GCLM - / - mice $(n=3)$. ROS were evaluated with CM-DCFDA by FACS analysis. (g) Basal respiration is increased in DIV7 cortical neurons derived from GCLM - / mice. Cortical neurons were analysed using the XF24 Analyser (see Materials and Methods section for details). (h) Western blotting analysis of the indicated proteins in DIV7 cortical neurons from GCLM - / - WT and Het mice. A representative experiment is shown. Syn, Synapsin 1-2

As shown in Figure 7f, DIV7 cortical neurons derived from GCLM - / - mice have higher levels of ROS and higher basal mitochondrial respiration (Figure $7 \mathrm{~g}$ ). However, no obvious differences were observed in mitochondrial function (Supplementary Figures S4c and d) and in neuronal differentiation assessed by the expression of Synapsin 1-2 and GAD67 (Figure 7h) between GCLM - / - and WT cortical neurons.

\section{Discussion}

Here we report for the first time a global metabolic profile in cortical neurons during the physiological process of terminal neuronal differentiation. Conversely, metabolic studies on neurons have mainly been conducted on pathological conditions, ${ }^{32-34}$ such as Parkinson's ${ }^{35}$ and Alzheimer's disease, ${ }^{36-38}$ or during cell death. ${ }^{39}$ Therefore, unraveling the metabolic changes underlying normal neuronal differentiation is an important baseline in which to interpret those metabolic changes associated with neurodegeneration. Although the model of in vitro terminal differentiation of cortical neurons has some limitations, we have chosen it to avoid any contribution from glia cell to the global metabolic profile of cortical neurons. ${ }^{40}$ Thus we show that the in vitro differentiation of primary cortical neurons is associated with increased glycolysis owing, at least in part, to increased expression of GLUT3 and of enzymes in the glycolytic pathway. Neuronal differentiation is also associated with increased glutamine metabolism, again at least partly owing to increased expression of enzymes such as GLS1 and GLS2, resulting in increased expression of neurotransmitters such as glutamate and GABA, and increased TCA cycle activity. Finally, differentiation requires a significant increased mitochondrial mass and function, secondary to activation of the PI3K/mTOR axis (Figure 8). Inhibition of these pathways arrests neuronal differentiation.

Our data suggest that the three metabolic changes we observe during differentiation have distinct roles at different 


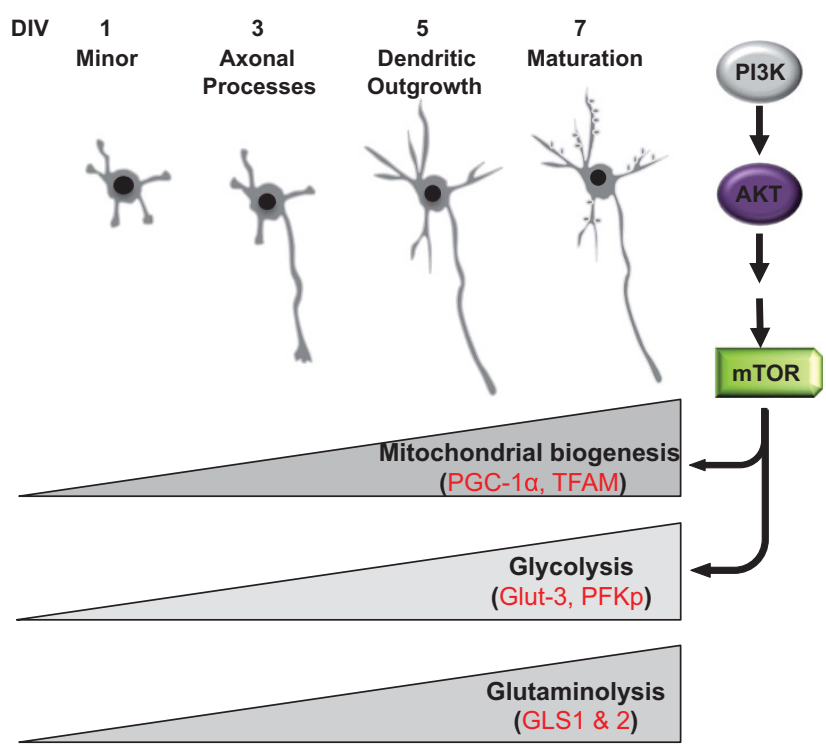

Figure 8 Schematic summary of the metabolic changes during terminal neuronal differentiation. Differentiation of primary cortical neurons is associated with increased mitochondrial biogenesis, glycolysis and glutaminolysis. Glycolysis is associated, at least in part, with an increased expression of GLUT3 and PFKp. Increased glutamine metabolism is partly due to an increased expression of enzymes, such as GLS1 and GLS2. Changes in mitochondrial mass, morphology and function were correlated with the upregulation of the master regulators of mitochondrial biogenesis, TFAM and PGC- $1 \alpha$. Differentiation requires a significant increased mitochondrial mass and function, secondary to activation of the PI3K/mTOR axis. Please see Discussion section for further details

stages of the differentiation process. The results indicate that glycolysis has a predominant role at an early phase of differentiation, whereas mitochondrial bioenergetics and glutamine are required for the later stages of differentiation, including dendritic outgrowth and maturation.

In neurons, mitochondria are involved in the regulation of $\mathrm{Ca}^{2+}$ homeostasis and redox signalling and in developmental, ${ }^{41}$ synaptic plasticity ${ }^{14,42}$ as well as in cell death. ${ }^{43-46}$ Previous reports have indicated a possible link between mitochondria and neuronal differentiation. Indeed, pharmacological inhibition of mitochondrial protein synthesis by CAF resulted in a reduction of differentiation, ${ }^{6}$ and mitochondrial morphology and movement changes with the development of mature synapses. ${ }^{5}$ In particular, it has been shown that PGC-1a, the master regulator of mitochondrial biogenesis, ${ }^{19,47,48}$ regulates the formation and maintenance of hippocampal synapses. ${ }^{49}$ Our findings not only confirm the role of mitochondria in neuronal differentiation but also add a further layer of complexity to mitochondrial biology in neurons. Thus, together with an increase in mitochondrial biogenesis, neuronal differentiation is associated with a reprogramming of mitochondrial structure and bioenergetics. In particular, mitochondria shift from a condensed matrix structure to the more orthodox structure having the typical cristae-rich morphology. ${ }^{50}$ This morphological shift is associated with functional changes, as the early-condensed DIV1 mitochondria operate near their maximal rate and have minimal spare respiratory capacity. In contrast, in the late phase of differentiation mitochondria have adopted a more classical configuration and have considerable spare respiratory capacity, indicating that mitochondria of fully differentiated cortical neurons are proportionally less active. Moreover, our in vitro findings are partially confirmed in vivo during postnatal development of the cortex, where we have observed an increase of both mtDNA and mitochondrial mass. However, further investigations are required for assessing the contribution of mitochondrial bioenergetics in neuronal differentiation both in embryos and in the developing brain.

Glucose is the main source of energy for the brain, ${ }^{51}$ and although the role of glycolysis in neurons is still debated, ${ }^{52}$ our findings indicate that, alongside its role in providing NADPH through the PPP pathway for regenerating GSH, glucose is crucially required for neuronal differentiation. Indeed, our results suggest that glucose is required to support the biosynthesis needed for neurite outgrowth and synaptic formation. This biosynthetic role is reminiscent of the Warburg effect observed in cancer cells and it has also been recently postulated in the rapidly growing human child brain. ${ }^{53}$ Moreover, ATP levels are reduced after 2-DG treatment, suggesting that glycolysis may be a major source of ATP early in the differentiation process, especially as it is only later that mitochondrial mass and function are increased. In agreement with our findings, more recently it has been shown that neurons uptake glucose and are the primary consumers of glucose. ${ }^{54}$

Glutamate is the main excitatory neurotransmitter in the brain and although there are many pathways for glutamate synthesis in cells, the vesicular pool in neurons is synthesized primarily from glutamine. ${ }^{25}$ Previously, it has also been shown that neurons under some circumstances can use glutamine and glutamate for energy production; ${ }^{3}$ our findings would suggest that this pathway is also involved in the regulation of neuronal differentiation. Indeed, pharmacological inhibition of the conversion of glutamine to glutamate leads to a reduction of neuronal differentiation and ATP production. This finding is also consistent with our previous observations that glutamine withdrawal from cortical neuron in culture reduces branching and the expression of synaptic proteins. ${ }^{55}$

Although the PI3K-Akt-mTOR pathway has emerged as a critical regulator of neuronal activity and morphology, ${ }^{27,56}$ here we show that pharmacological inhibition of these pathways resulted in a reduction of mitochondrial biogenesis and glucose metabolism in neurons. Therefore, our findings not only support the published role of mTOR signalling in the regulation of dendritic arborization, ${ }^{11}$ but also suggest that increased mitochondrial mass and function, together with enhanced glycolysis may be required for dendrite and synapse formation. As mTOR regulates mitochondrial biogenesis via TFAM, ${ }^{57}$ our data would emphasize the central critical role of $\mathrm{mTOR}$ in neuronal differentiation.

\section{Materials and Methods}

Mice. Mice were bred and subjected to listed procedures under the Project Licence PPL 40/3442 released from the UK Home Office. GCLM - / - mice were generated as previously described. ${ }^{30,26}$

Cortical neuron preparation and treatment. Primary cortical neuronal were prepared from E17.5 embryos mouse as previously described. ${ }^{23}$ Briefly, cortices were harvested and cut into smaller pieces. Tissue was incubated with $1 \times$ Trypsin (Invitrogen, Loughborough, UK) for $10 \mathrm{~min}$ at $37^{\circ} \mathrm{C}$. After washing with Neurobasal medium (Invitrogen) containing 10\% FCS, penicillin (100 U), streptomycin $(100 \mu \mathrm{g})$ (Invitrogen), and 1X Glutamax (Invitrogen), tissue was 
resuspended by gentle pipetting. Cells were diluted to the appropriate concentration and then plated in aforementioned medium on poly-D-lysine-coated plates. After $1 \mathrm{~h}$, medium was replaced with Neurobasal medium (Invitrogen) containing B27 supplement (Invitrogen), penicillin (100U), streptomycin (100 $\mu \mathrm{g})$ (Invitrogen) and 1X Glutamax (Invitrogen) and maintained in culture for different days in vitro. Cortical neurons were treated with the following drugs as indicated in figure legend.

2-DG, DON, ${ }^{26} \mathrm{CAF}$ and $\mathrm{NAC}^{30}$ were purchased from Sigma-Aldrich (Dorset, UK). Rapamycin, LY294002 and PD98059 were purchased from Cell Signaling (Hitchin, UK).

Metabolic profiling. Samples were immediately stored at $-80^{\circ} \mathrm{C}$. Samples were extracted and prepared for analysis using Metabolon's standard solvent extraction method. The sample preparation process was carried out using the automated MicroLab STAR system from Hamilton Company (Reno, NV, USA). Recovery standards were added prior to the first step in the extraction process for QC purposes. Sample preparation was conducted using a proprietary series of organic and aqueous extractions to remove the protein fraction while allowing maximum recovery of small molecules. Then the resulting extract was divided into two fractions; one for analysis by liquid chromatography (LC) and the other one for analysis by gas chromatography. Samples were placed briefly on a TurboVap (Zymark, Hopkinton, MA, USA) to remove the organic solvent. Each sample was then frozen and dried under vacuum. Samples were then prepared for the appropriate instrument, either LC/mass spectrometry (MS) or GC/MS. Instrument variability was determined by calculating the median relative standard deviation (RSD) for the internal standards that were added to each sample prior to injection into the mass spectrometers. Overall process variability was determined by calculating the median RSD for all endogenous metabolites (i.e., non-instrument standards) present in $100 \%$ of the Client Matrix samples, which are technical replicates of pooled samples. Following normalization to total protein determined by Bradford assay, log transformation and imputation with minimum observed values was performed for each compound. Welch's two-sample $t$-tests were then used to identify biochemicals that differed significantly between experimental groups. A summary of the numbers of biochemicals that achieved statistical significance $(P \leq 0.05)$ as well as those approaching significance $(0.05<P<0.1)$, is shown.

Transmission electron microscopy. For electron microscopy, cells were fixed in $2 \%$ glutaraldehyde in $0.1 \mathrm{M}$ sodium cacodylate buffer $(\mathrm{pH} 7.4)$ at $4{ }^{\circ} \mathrm{C}$ overnight and postfixed with $1 \%$ osmium tetroxide/ $1 \%$ potassium ferrocyanide for $1 \mathrm{~h}$ at room temperature. After fixation, cells were stained en bloc with $5 \%$ aqueous uranyl acetate overnight at room temperature, dehydrated and embedded in Taab epoxy resin (Taab Laboratories Equipment Ltd, Aldermaston, UK). Ultrathin sections were stained with lead citrate and recorded using a Megaview 3 digital camera and iTEM software (Olympus Soft Imaging Solutions GmbH, Münster, Germany) in a Jeol 100-CXII electron microscope (Jeol UK Ltd, Welwyn Garden City, UK).

Extracellular flux (XF) analysis. Cortical neurons were seeded in XF 24well cell culture microplate (Seahorse Bioscience, Copenhagen, Denmark) in quintuplicate at $3 \times 10^{5}$ cells/well in $1 \mathrm{ml}$ growth medium and then incubated at $37^{\circ}$ $\mathrm{C}$ in $5 \% \mathrm{CO}_{2}$. Cortical neurons from GCLM - / - mice were seeded in XF 96-well cell culture microplate (Seahorse Bioscience) in quintuplicate at $8 \times 10^{4}$ cells/well. Assays were initiated by removing the growth medium from each well and replacing it with 600-900 $\mu$ l of assay medium prewarmed to $37^{\circ} \mathrm{C}$. The cells were incubated at $37^{\circ} \mathrm{C}$ for $30 \mathrm{~min}$ to allow media temperature and $\mathrm{pH}$ to reach equilibrium before the first rate measurement. Prior to each rate measurement, the XF24 Analyser gently mixed the assay media in each well for $10 \mathrm{~min}$ to allow the oxygen partial pressure to reach equilibrium. Following mixing, OCR and ECAR were measured simultaneously for 3-5 min to establish a baseline rate. The assay medium was then gently mixed again for 3-5 min between each rate measurement to restore normal oxygen tension and $\mathrm{pH}$ in the microenvironment surrounding the cells. After the baseline measurement, 75-90 $\mu \mathrm{l}$ of a testing agent prepared in assay medium was then injected into each well to reach the desired final working concentration. This was followed by mixing for 5-10 min to expedite compound exposure to cellular proteins, after which OCR and ECAR measurements were then made. Generally, two to three baseline rates and two or more response rates (after compound addition) were measured, and the average of two baseline rates or test rates was used for data analysis. For time-resolved experiments, multiple measurements as well as compound injections were made at the time points indicated. The values of OCR and ECAR reflect both the metabolic activities of the cells and the number of cells being measured. Typically, at the end of each assay cells are treated for protein extraction.

RNA extraction and real-time PCR. Total RNA from cells was isolated using Trizol (Invitrogen) according to the manufacturer's instructions. Total RNA $(3 \mu \mathrm{g})$ was reverse transcribed using RevertAid H Minus Reverse Transcriptase and oligo(dT) (Thermo Scientific, Loughbrough, UK). qRT-PCR was performed in an ABI PRISM 7000 Sequence Detection System (Applied Biosystem, Loughbrough, UK) with SYBR green ready mix (Applied Biosystem) and specific primers. The expression of each gene was defined from the threshold cycle, and the relative expression levels were calculated by using the $2^{-\Delta \Delta C t}$ method. The following primers were used:

HK1 Fwd 5'-GTGGACGGGACGCTCTAC-3',

HK1 Rev 5'-TTCACTGTTTGGTGCATGATT-3',

PKM2 Fwd 5'-GCCGCCTGGACATTGACTC-3',

PKM2 Rev 5'-CCATGAGAGAAATTCAGCCGAG-3',

PFKp Fwd 5'-GGAAGCCAAATGGGACTGT-3',

PFKp Rev 5'-CGCACTACCGATGATGGTC-3',

GLUD1 Fwd 5'-CCCAACTTCTTCTTCAAGATGGTGG-3',

GLUD1 Rev 5'-AGAGGCTCAACACATGGTTGC-3',

GLS1 Fwd 5'-GGGAATTCACTTTTGTCACGA-3',

GLS1 Rev 5'-GACTTCACCCTTTGATCACC-3',

GLS2 Fwd 5'-AGCGTATCCCTATCCACAAGTTCA-3',

GLS2 Rev 5'-GCAGTCCAGTGGCCTTCAGAG-3',

GLUT3 Fwd 5'-ATGGGGACAACGAAGGTGAC-3',

GLUT3 Rev 5'-GTCTCAGGTGCATTGATGACTC-3',

GADPH Fwd 5'-CAATGAATACGGCTACAGCAAC-3',

GADPH Rev 5'-AGGGAGATGCTCAGTGTTGG-3',

GPX1 Fwd 5'-CAGGAGAATGGCAAGAATGA-3',

GPX1 Rev 5'-GAAGGTAAAGAGCGGGTGAG-3',

SOD1 Fwd 5'-GAGCCGGCTTGGCATCCGTT-3',

SOD1 Rev 5'-CGCGTCCTGCGGCCTTAGTC-3',

COX2 Fwd 5'-TCTCCCCTCTCTACGCATTC-3',

COX2 Rev 5'-CAGGTTTTAGGTCGTTTGTTG-3',

TFB2M Fwd 5'-AAGATGGCCCTTTCGTTTATGG-3', and

TFB2M Rev 5'-GACTGTGCTGTTTGCTTCCTG-3'.

ROS levels. Cortical neurons were trypsinized and $\mathrm{CM}-\mathrm{H}_{2} \mathrm{DCFDA}$ (Invitrogen) was added at the final concentration of $10 \mu \mathrm{M}$. Cells were incubated for $20 \mathrm{~min}$ at $37^{\circ} \mathrm{C}$ to allow the permeabilization of the probes and were analysed by flow cytometry acquiring 15000 events per sample. In detail, the $\mathrm{CM}-\mathrm{H}_{2} \mathrm{DCFDA}$ fluorescent signal was collected in the FL1-H channel.

Glucose uptake. Glucose uptake was monitored using fluorescent 2-NBDG (Invitrogen). Before staining cortical neurons with 2-NBDG, culture medium was removed and the cells were incubated with fresh culture medium containing $2 \mathrm{mM}$ glucose for $15 \mathrm{~min}$. Then fluorescent 2-NBDG was added at final concentration of $50 \mu \mathrm{M}$ and cells were incubated at $37^{\circ} \mathrm{C}$ with $5 \% \mathrm{CO}_{2}$ for $1 \mathrm{~h}$. 2-NBDG uptake were stopped by removing the incubation medium and washing the cells with prewarmed culture medium ( $2 \mathrm{mM}$ glucose), harvested, centrifuged and subsequently suspended in prewarmed culture medium ( $2 \mathrm{mM}$ glucose). The samples were then analysed using a FACScan flow cytometer (Becton Dickinson, Oxford, UK).

mtDNA relative quantification. Cortical neurons were washed with prewarmed PBS and harvested. The cell pellet were suspended with $600 \mu \mathrm{l}$ of extraction buffer $(\mathrm{NaCl} 75 \mathrm{mM}, 50 \mathrm{mM}$ Tris $\mathrm{HCl}$ pH 8.5, EDTA $5 \mathrm{mM}$, SDS $1 \%$, Proteinase $\mathrm{K} 500 \mu \mathrm{g} / \mathrm{ml}$ ) and incubated for $2 \mathrm{~h}$ at $55^{\circ} \mathrm{C}$. Then samples were incubated for $10 \mathrm{~min}$ at $95^{\circ} \mathrm{C}$ to inhibit the Proteinase $\mathrm{K}$ and centrifuged at 8000 r.p.m. for $15 \mathrm{~min}$ at $4^{\circ} \mathrm{C}$. Supernatant was recovered and the same volume of phenol/ chloroform/isoamyl alcohol $(25: 4: 1)$ was added. After vortexing and centrifugation at 13000 r.p.m. for $15 \mathrm{~min}$ at $4^{\circ} \mathrm{C}$, the aqueous phase was recovered and DNA was precipitated with $1 / 10 \mathrm{NaAc}(3 \mathrm{M}, \mathrm{pH}$ 5.2) and 1 volume of isopropanol for $10 \mathrm{~min}$ at $-20^{\circ} \mathrm{C}$. After centrifugation, the DNA pellet was washed with $70 \% \mathrm{EtOH}$, air dried and dissolved in sterile water. DNA concentrations were measured using a Nanodrop 1000 spectrometer (Thermo Scientific). The relative amount of mtDNA was valued by qRT-PCR performed in an ABI PRISM 7000 Sequence Detection System (Applied Biosystem) with SYBR green ready mix (Applied Biosystem) with the following primers: 
SDHA Fwd 5'-AGACCTTGAATGAGGCTGACTGTG-3', SDHA Rev 5'-GAGACAAAGTCTGGCGCAACTC-3', ND5 Fwd 5'-TGATGGTACGGACGAACAGACG-3', and ND5 Rev 5'-CTGATGGTAGTCATGGGTGGAG-3'

ATP levels. Intracellular levels of ATP were measured by using the ELITEN ATP Assay System Bioluminescence Detection Kit (Promega, Southampton, UK). Briefly, treated and untreated cells were harvested and processed according to the manufacturer's instructions. The total levels of ATP were normalized to the protein content.

Western blotting. Proteins were extracted with RIPA buffer containing an inhibitor cocktail (Roche, Sussex, UK) and protein concentration was determined using a Bradford dye-based assay (Bio-Rad, Hemel Hempstead, UK). Total protein $(30 \mu \mathrm{g})$ was subjected to SDS-PAGE followed by immunoblotting with appropriate antibodies at the recommended dilutions. The blots were then incubated with peroxidase-linked secondary antibodies followed by enhanced-chemiluminescent detection using the Super Signal Chemiluminescence Kit (Thermo scientific). The following antibodies were used: PGC-1 $\alpha$ (rabbit, $1: 1000$, Abcam, Cambridge, UK), MEF2 (rabbit, 1:500, Santa Cruz, Middlesex, UK), Phospho-S6 Ribosomal Protein (Ser235/236) (rabbit, $1: 100$, Cell Signaling Technology, UK), S6 Ribosomal Protein (5G10) (Rabbit, 1:100, Cell Signaling Technology), TFAM (rabbit, $1: 100$, Sigma, UK), NRF1 (rabbit, 1:500, Life Span Bioscience, Nottingham, UK), Cleaved caspase-3 (rabbit, 1:1 000, Cell Signaling), GAD67 (mouse, 1:1000, Millipore, Watford, UK), MitoProfile Total OXPHOS Rodent WB Antibody Cocktail (mouse, $1: 250$, Abcam, UK), LC3B (rabbit, $1: 1000$, Sigma), $\beta$-Tubulin (rabbit, $1: 1000$, Santa Cruz), Synapsin (1:1000, Synaptic System, Goettingen, Germany), Actin (1: 5000, Sigma), Cyclophillin D (1: 1000, Abcam), GLS2 (1: 100, Abcam), PFKp (1:500, Abcam) and GADPH (mouse, $1: 10000$, Sigma).

Immunofluorescence. Cortical neurons were fixed with 3\% paraformaldehyde in PBS followed by treatment with $0.1 \%$ Triton X-100 for 5 min and $10 \%$ normal goat serum in PBS for $1 \mathrm{~h}$ at RT. Then cells were stained at $4{ }^{\circ} \mathrm{C}$ overnight with the following primary antibodies: neuronal-specific anti- $\beta$-III-Tubulin ( $1: 2000$; Promega). After three washes (10 min, PBS), nuclei were stained with DAPI $(1: 10000)$ for $10 \mathrm{~min}$; this step was followed by a further wash (10 min in PBS). The coverslips were then mounted with Aquapolymount antifading solution (Polysciences, Northampton, UK) onto glass slides and observed under a confocal microscope (Zeiss LSM 510, Cambridge, UK)

Statistical analysis. All results are expressed as means \pm S.D. $P<0.05$ was considered significant. Welch's two-sample $t$-tests were then used to identify biochemicals that differed significantly between the experimental groups. Statistical analysis were performed using GraphPad Prism 6 (La Jolla, CA, USA).

\section{Conflict of Interest}

The authors declare no conflict of interest.

Acknowledgements. We thank Tim Smith and Maria Guerra Martin for their technical support in transmission electron microscopy. We thank Professor Kelvin Cain for his assistance in the Seahorse experiments. We thank the staff members of the animal facility at University of Leicester. We thank Professor Alessandro FinazziAgro' and Dr. Ivano Amelio for scientific discussion. This work has been supported by the Medical Research Council, UK. This work was supported by AIRC IG grant (2014-IG15653), AIRC5xmille grant (2010-MCO no. 9979) and Fondazione Roma NCDs grant awarded to GM.

1. Attwell $D$, Laughlin SB. An energy budget for signaling in the grey matter of the brain. J Cereb Blood Flow Metab 2001; 21: 1133-1145.

2. McKenna MC. The glutamate-glutamine cycle is not stoichiometric: fates of glutamate in brain. J Neurosci Res 2007; 85: 3347-3358.

3. Erecinska M, Zaleska MM, Nissim I, Nelson D, Dagani F, Yudkoff M. Glucose and synaptosomal glutamate metabolism: studies with [15 Ngglutamate. J Neurochem 1988; 51: 892-902.

4. Sonnewald U, McKenna M. Metabolic compartmentation in cortical synaptosomes: influence of glucose and preferential incorporation of endogenous glutamate into GABA. Neurochem Res 2002; 27: 43-50.

5. Chang DT, Reynolds IJ. Differences in mitochondrial movement and morphology in young and mature primary cortical neurons in culture. Neuroscience 2006; 141: 727-736.
6. Vayssiere JL, Cordeau-Lossouarn L, Larcher JC, Basseville M, Gros F, Croizat B. Participation of the mitochondrial genome in the differentiation of neuroblastoma cells. In Vitro Cell Dev Biol 1992; 28A: 763-772.

7. de la Torre-Ubieta L, Bonni A. Transcriptional regulation of neuronal polarity and morphogenesis in the mammalian brain. Neuron 2011; 72: 22-40.

8. Kristiansen M, Ham J. Programmed cell death during neuronal development: the sympathetic neuron model. Cell Death Differ 2014; 21: 1025-1035.

9. Avila A, Vidal PM, Tielens S, Morelli G, Laguesse S, Harvey RJ et al. Glycine receptors control the generation of projection neurons in the developing cerebral cortex. Cell Death Differ 2014; 21: 1696-1708.

10. Dotti $C G$, Sullivan $C A$, Banker GA. The establishment of polarity by hippocampal neurons in culture. J Neurosci 1988; 8: 1454-1468.

11. Kumar V, Zhang MX, Swank MW, Kunz J, Wu GY. Regulation of dendritic morphogenesis by Ras-PI3K-Akt-mTOR and Ras-MAPK signaling pathways. J Neurosci 2005; 25: 11288-11299.

12. Urbanska M, Gozdz A, Swiech LJ, Jaworski J. Mammalian target of rapamycin complex 1 (mTORC1) and 2 (mTORC2) control the dendritic arbor morphology of hippocampal neurons. J Biol Chem 2012; 287: 30240-30256.

13. Onyango IG, Lu J, Rodova M, Lezi E, Crafter AB, Swerdlow RH. Regulation of neuron mitochondrial biogenesis and relevance to brain health. Biochim Biophys Acta 2010; 1802: 228-234.

14. Mattson MP, Gleichmann M, Cheng A. Mitochondria in neuroplasticity and neurological disorders. Neuron 2008; 60: 748-766.

15. Gioran A, Nicotera P, Bano D. Impaired mitochondrial respiration promotes dendritic branching via the AMPK signaling pathway. Cell Death Dis 2014; 5: e1175.

16. Fletcher TL, Banker GA. The establishment of polarity by hippocampal neurons: the relationship between the stage of a cell's development in situ and its subsequent development in culture. Dev Biol 1989; 136: 446-454.

17. Hackenbrock CR. Ultrastructural bases for metabolically linked mechanical activity in mitochondria. II. Electron transport-linked ultrastructural transformations in mitochondria. J Cell Biol 1968; 37: 345-369.

18. Medeiros DM. Assessing mitochondria biogenesis. Methods 2008; 46: 288-294.

19. Lin J, Handschin C, Spiegelman BM. Metabolic control through the PGC-1 family of transcription coactivators. Cell Metab 2005; 1: 361-370.

20. Heidenreich KA, Linseman DA. Myocyte enhancer factor-2 transcription factors in neuronal differentiation and survival. Mol Neurobiol 2004; 29: 155-166.

21. Johar $\mathrm{K}$, Priya A, Wong-Riley MT. Regulation of $\mathrm{Na}(+) / \mathrm{K}(+)-$ ATPase by nuclear respiratory factor 1: implication in the tight coupling of neuronal activity, energy generation, and energy consumption. J Biol Chem 2012; 287: 40381-40390.

22. St-Pierre J, Lin J, Krauss S, Tarr PT, Yang R, Newgard CB et al. Bioenergetic analysis of peroxisome proliferator-activated receptor gamma coactivators 1alpha and 1beta (PGC-1alpha and PGC-1beta) in muscle cells. J Biol Chem 2003; 278: 26597-26603.

23. Agostini M, Tucci P, Killick R, Candi E, Sayan BS, Rivetti di Val Cervo P et al. Neuronal differentiation by TAp73 is mediated by microRNA-34a regulation of synaptic protein targets. Proc Natl Acad Sci USA 2011; 108: 21093-21098.

24. Nehlig A, Coles JA. Cellular pathways of energy metabolism in the brain: is glucose used by neurons or astrocytes? Glia 2007; 55: 1238-1250.

25. Marquez J, Tosina M, de la Rosa V, Segura JA, Alonso FJ, Mates JM et al. New insights into brain glutaminases: beyond their role on glutamatergic transmission. Neurochem Int 2009; 55: 64-70.

26. Harris IS, Treloar AE, Inoue S, Sasaki M, Gorrini C, Lee KC et al. Glutathione and thioredoxin antioxidant pathways synergize to drive cancer initiation and progression. Cancer Cell 2015; 27: 211-222.

27. Jaworski J, Sheng M. The growing role of $\mathrm{mTOR}$ in neuronal development and plasticity. Mo Neurobiol 2006; 34: 205-219

28. Laplante M, Sabatini DM. mTOR signaling in growth control and disease. Cell 2012; 149: 274-293.

29. Vieira HL, Alves PM, Vercelli A. Modulation of neuronal stem cell differentiation by hypoxia and reactive oxygen species. Progr Neurobiol 2011; 93: 444-455.

30. McConnachie LA, Mohar I, Hudson FN, Ware CB, Ladiges WC, Fernandez C et al. Glutamate cysteine ligase modifier subunit deficiency and gender as determinants of acetaminophen-induced hepatotoxicity in mice. Toxicol Sci 2007; 99: 628-636.

31. Steullet P, Cabungcal JH, Kulak A, Kraftsik R, Chen Y, Dalton TP et al. Redox dysregulation affects the ventral but not dorsal hippocampus: impairment of parvalbumin neurons, gamma oscillations, and related behaviors. J Neurosci 2010; 30: 2547-2558.

32. Narayanan SP, Xu Z, Putluri N, Sreekumar A, Lemtalsi T, Caldwell RW et al. Arginase 2 deficiency reduces hyperoxia-mediated retinal neurodegeneration through the regulation of polyamine metabolism. Cell Death Dis 2014; 5: e1075.

33. Krug AK, Gutbier S, Zhao L, Poltl D, Kullmann C, Ivanova V et al. Transcriptional and metabolic adaptation of human neurons to the mitochondrial toxicant MPP(+). Cell Death Dis 2014; 5: e1222.

34. Brustovetsky N. Mutant Huntingtin and elusive defects in oxidative metabolism and mitochondrial calcium handling. Mol Neurobiol 2015 (e-pub ahead of print).

35. Pickrell AM, Youle RJ. The roles of PINK1, parkin, and mitochondrial fidelity in Parkinson's disease. Neuron 2015; 85: 257-273.

36. Burte F, Carelli V, Chinnery PF, Yu-Wai-Man P. Disturbed mitochondrial dynamics and neurodegenerative disorders. Nat Rev Neurol 2015; 11: 11-24. 
37. Soucek T, Cumming R, Dargusch R, Maher P, Schubert D. The regulation of glucose metabolism by HIF-1 mediates a neuroprotective response to amyloid beta peptide. Neuron 2003; 39: 43-56.

38. Ray A, Martinez BA, Berkowitz LA, Caldwell GA, Caldwell KA. Mitochondrial dysfunction, oxidative stress, and neurodegeneration elicited by a bacterial metabolite in a $C$. elegans Parkinson's model. Cell Death Dis 2014; 5: e984.

39. Almeida A, Moncada S, Bolanos JP. Nitric oxide switches on glycolysis through the AMP protein kinase and 6-phosphofructo-2-kinase pathway. Nat Cell Biol 2004; 6: 45-51.

40. Magistretti PJ. Neuron-glia metabolic coupling and plasticity. Exp Physiol 2011; 96: 407-410.

41. Bonora M, De Marchi E, Patergnani S, Suski JM, Celsi F, Bononi A et al. Tumor necrosis factor-alpha impairs oligodendroglial differentiation through a mitochondria-dependent process. Cell Death Differ 2014; 21: 1198-1208.

42. Kann O, Kovacs R. Mitochondria and neuronal activity. Am J Physiol Cell Physiol 2007; 292 : C641-C657.

43. Kim JE, Ryu HJ, Kim MJ, Kang TC. LIM kinase-2 induces programmed necrotic neuronal death via dysfunction of DRP1-mediated mitochondrial fission. Cell Death Differ 2014; 21: 1036-1049.

44. Hwang MS, Schwall CT, Pazarentzos E, Datler C, Alder NN, Grimm S. Mitochondrial Ca(2+) influx targets cardiolipin to disintegrate respiratory chain complex II for cell death induction. Cell Death Differ 2014; 21: 1733-1745.

45. Sisalli MJ, Secondo A, Esposito A, Valsecchi V, Savoia C, Di Renzo GF et al. Endoplasmic reticulum refilling and mitochondrial calcium extrusion promoted in neurons by NCX1 and NCX3 in ischemic preconditioning are determinant for neuroprotection. Cell Death Differ 2014; 21: 1142-1149.

46. Cavallucci V, Bisicchia E, Cencioni MT, Ferri A, Latini L, Nobili A et al. Acute focal brain damage alters mitochondrial dynamics and autophagy in axotomized neurons. Cell Death Dis 2014; 5: e1545.

47. Baldelli S, Aquilano K, Ciriolo MR. PGC-1alpha buffers ROS-mediated removal of mitochondria during myogenesis. Cell Death Dis 2014; 5: e1515.

48. Chen W, Wang Q, Bai L, Chen W, Wang X, Tellez CS et al. RIP1 maintains DNA integrity and cell proliferation by regulating PGC-1alpha-mediated mitochondrial oxidative phosphorylation and glycolysis. Cell Death Differ 2014; 21: 1061-1070.

49. Cheng A, Wan R, Yang JL, Kamimura N, Son TG, Ouyang $X$ et al. Involvement of $P G C-1$ alpha in the formation and maintenance of neuronal dendritic spines. Nat Commun 2012; 3: 1250.
50. Hackenbrock CR. Ultrastructural bases for metabolically linked mechanical activity in mitochondria. I. Reversible ultrastructural changes with change in metabolic steady state in isolated liver mitochondria. J Cell Biol 1966; 30: 269-297.

51. Peters A, Schweiger U, Pellerin L, Hubold C, Oltmanns KM, Conrad M et al. The selfish brain: competition for energy resources. Neurosci Biobehav Rev 2004; 28: 143-180.

52. Bolanos JP, Almeida A, Moncada S. Glycolysis: a bioenergetic or a survival pathway? Trends Biochem Sci 2010; 35: 145-149.

53. Goyal MS, Hawrylycz M, Miller JA, Snyder AZ, Raichle ME. Aerobic glycolysis in the human brain is associated with development and neotenous gene expression. Cell Metab 2014; 19: $49-57$.

54. Lundgaard I, Li B, Xie L, Kang H, Sanggaard S, Haswell JD et al. Direct neuronal glucose uptake heralds activity-dependent increases in cerebral metabolism. Nat Commun 2015; 6 : 6807.

55. Velletri T, Romeo F, Tucci P, Peschiaroli A, Annicchiarico-Petruzzelli M, Niklison-Chirou MV et al. GLS2 is transcriptionally regulated by $\mathrm{p} 73$ and contributes to neuronal differentiation. Cell Cycle 2013; 12: 22.

56. Liu Q, Qiu J, Liang M, Golinski J, van Leyen K, Jung JE et al. Akt and mTOR mediate programmed necrosis in neurons. Cell Death Dis 2014; 5: e1084.

57. Morita M, Gravel SP, Chenard V, Sikstrom K, Zheng L, Alain T et al. mTORC1 controls mitochondrial activity and biogenesis through $4 \mathrm{E}-\mathrm{BP}$-dependent translational regulation. Cell Metab 2013; 18: 698-711.

(c) (i) $(\Theta)$ This work is licensed under a Creative Commons Attribution-NonCommercial-NoDerivs 4.0 International License. The images or other third party material in this article are included in the article's Creative Commons license, unless indicated otherwise in the credit line; if the material is not included under the Creative Commons license, users will need to obtain permission from the license holder to reproduce the material. To view a copy of this license, visit http://creativecommons.org/licenses/by-nc-nd/4.0/

(C) The Author(s) 2016

Supplementary Information accompanies this paper on Cell Death and Differentiation website (http://www.nature.com/cdd) 